\title{
A 1.5D Spectral Kurtosis-Guided TQWT Method and Its Application in Bearing Fault Detection
}

\author{
Xiong Zhang $\left(\mathbb{D},{ }^{1}\right.$ Ming Zhang, ${ }^{1}$ Shuting Wan, ${ }^{1}$ Rujiang Hao $\mathbb{D}^{\circ},{ }^{2}$ Yuling He, \\ and Xiaolong Wang ${ }^{1}$ \\ ${ }^{1}$ Hebei Key Laboratory of Electric Machinery Health Maintenance \& Failure Prevention, North China Electric Power University, \\ Baoding 071003, China \\ ${ }^{2}$ State Key Laboratory of Mechanical Behavior and System Safety of Traffic Engineering Structures, \\ Shijiazhuang Tiedao University, Shijiazhuang 050043, China
}

Correspondence should be addressed to Rujiang Hao; hdjxzx1990@163.com

Received 21 January 2021; Revised 9 April 2021; Accepted 3 May 2021; Published 17 May 2021

Academic Editor: Fabio Minghini

Copyright (C) 2021 Xiong Zhang et al. This is an open access article distributed under the Creative Commons Attribution License, which permits unrestricted use, distribution, and reproduction in any medium, provided the original work is properly cited.

\begin{abstract}
Bearings are the key parts of rotating machinery, and their operation status is related to the operation safety of the whole equipment. Vibration signals often contain periodic impulse components which can reflect the fault state of bearings. However, due to the interference of signal transmission path and the influence of operating environment noise, the periodic impulse components in the signal are often submerged by the nonperiodic transient impulse components, modulation harmonic components, and noise components. Therefore, the core problem of bearing fault diagnosis theory is used to accurately extract the frequency band of bearing fault state information and suppress the frequency band of interference information. In this paper, the signal is processed by the tunable Q-factor wavelet transform (TQWT), the midfrequency band of the signal is tightly divided by selecting different Q-values, and the 1.5D spectral kurtosis defined in frequency domain is used to select the optimal subband. Simulated analysis shows that this method can avoid low-frequency harmonic interference, nonperiodic transient impulse components, and strong noise components in the time domain. Therefore, it can effectively realize the selection of the subbands of periodic impulse components and effectively extract fault feature information. Through experimental signal analysis, TQWT has good sparsity decomposition characteristics and can reasonably divide the signal frequency band, so as to separate the useful fault characteristic frequency band and interference frequency band. At the same time, compared with the kurtosis index, 1.5D spectral kurtosis has better robustness and resolution for low signal-to-noise ratio signals, which can achieve the purpose of fault characteristic band extraction.
\end{abstract}

\section{Introduction}

Rolling bearing is an important component of rotating machinery, and its operation status is directly related to whether the whole equipment can run safely and smoothly. Therefore, the fault analysis of rolling bearing has become a hot issue in the field of signal analysis [1-3]. Considering the problems of signal transmission path, signal acquisition convenience, and cost, vibration signal is a widely used analysis medium. So many analysis and processing methods based on vibration signal are derived [4-6]. Especially, in recent years, with the development of mathematical model construction and parameter optimization method of processing algorithm [7-9], signal processing algorithm has been greatly improved in terms of accuracy, efficiency, and adaptability.

The key link of bearing vibration signal processing is filtering process. Through certain analysis methods, the fault information within the specific frequency band can be retained to the maximum extent, and the interference components outside the frequency band can be suppressed to the maximum extent, so as to realize the signal filtering and fault information extraction. There are two main problems: one is to use appropriate methods to segment the 
frequency band, which makes the subband after segmentation have certain sparsity, and the other is to use appropriate index to evaluate the subband after segmentation, so that the subband with fault impulse component has certain specificity. The kurtogram algorithm [10] proposed by Antoni is considered to be a typical signal processing method, which can divide the signal plane into subbands with different bandwidth and center frequency through filter banks and select the subbands by the kurtosis index. Subsequently, many scholars began to improve the frequency band segmentation process and index selection of this kind of algorithm. Lei et al. [11] used wavelet packet decomposition to replace the equivalent filter banks in the kurtogram algorithm. Wang and Tsui [12] used dynamic Bayes to optimize the wavelet parameters and adjust the center frequency and bandwidth of the equivalent filtering characteristics, so that it can more accurately locate the fault impact subband. Qin et al. [13] constructed a new M-band flexible wavelet transform to identify the potential fault features in the measured signal. This transform provides more intensive time-frequency plane sampling and retains the tunable filter parameters and dilation factors. The simulated and experimental results show that this transform has better denoising performance than analytic flexible wavelet transform, orthogonal wavelet transform, and biorthogonal wavelet transform. Meanwhile, Qin et al. [14] proposed a novel K-SVD algorithm to get the optimized transient dictionary for the one-dimensional signal. Simulated and experimental results show that this method has advantages over the traditional K-SVD method and infogram method in periodic transient impulse components detection. The kurtosis index is too sensitive to impulse components and is easily disturbed by nonperiodic transient impulses and noise. Based on these considerations, Antoni further defines the square envelope entropy index and the square envelope spectral entropy index in the time domain and frequency domain, respectively, and constructs the infogram algorithm [15]. Li et al. [16] proposed a multiscale clustering gray infogram, which combined two negative entropies with the gray method. Moshrefzadeh and Fasana [17] proposed an autogram method using square envelope unbiased autocorrelation instead of time-domain signal. Through this operation, uncorrelated interference components can be discarded.

Selesnick designed the tunable Q-factor wavelet transform (TQWT) from the perspective of frequency domain filtering [18]. TQWT can make the sparsity of frequency band division more diversified by adjusting its Q-factor. The key problem of TQWT is how to determine its parameter value and make the selection of frequency band more accurate by adjusting the oscillation characteristics of wavelet. Zhao et al. [19] used the particle swarm optimization algorithm to optimize TQWT parameters, combined with the spectral kurtosis index to select the optimal subband. $\mathrm{Ma}$ et al. [20] also used particle swarm optimization to optimize the parameters of TQWT and further combined with frequency slice wavelet transform (FSWT) to select the frequency band. Ding et al. [21] constructed a filter bank with almost constant bandwidth by adjusting the Q-factor and decomposition level of TQWT.

In this paper, a $1.5 \mathrm{D}$ spectral kurtosis-guided TQWT method is proposed. In this method, the $1.5 \mathrm{D}$ spectral kurtosis of the square envelope sequence of wavelet subband signals with different Q-values and scales is calculated to replace the traditional kurtosis index to select the optimal subband. This method can suppress the noise and the nonperiodic transient impulse interference without coupling relationship.

The outline of this paper is as follows. Section 2 introduces the 1.5D spectral kurtosis-guided TQWT method and its superiority in signal periodic feature extraction. Sections 3 and 4 verify the effectiveness of the proposed method with the aid of simulated and experimental data.

\section{1.5D Spectral Kurtosis-Guided TQWT}

2.1. TQWT. Compared with the traditional discrete wavelet transform, TQWT can determine the wavelet basis function by designing the Q-factor (the Q-factor is the ratio of signal center frequency to bandwidth, $\left.Q=f_{c} / B_{\omega}\right)$ and the redundancy factor according to the signal oscillation characteristics. This design makes the choice of wavelet function more flexible, makes the signal adaptability stronger, and makes it easier to control the sparsity of frequency band division. TQWT uses band-pass filter banks to realize signal decomposition and reconstruction in an iterative manner. The three-level decomposition and reconstruction is taken as an example. The corresponding dual channel filter banks are shown in Figure 1.

In Figure 1, $H_{1}(\omega)$ and $H_{0}(\omega)$ are the frequency response functions of the high-pass filter and the low-pass filter $\left(H_{1}(\omega)\right.$ and $H_{0}(\omega)$ can be expressed as equations (1) and $(2)), H_{1}^{*}(\omega)$ and $H_{0}^{*}(\omega)$ are the conjugate complex numbers of $H_{1}(\omega)$ and $H_{0}(\omega)$, respectively, $\beta=2 /(Q+1)$ is the high-pass scaling factor, and $\alpha=1-\beta / r$ is the low-pass scaling factor, where $r$ is the redundancy factor:

$$
\begin{aligned}
& H_{0}(\omega)= \begin{cases}1, & |\omega| \leq(1-\beta), \\
\theta\left[\frac{\omega+(\beta-1) \pi}{\alpha+\beta-1}\right], & (1-\beta) \pi \leq \omega<\alpha \pi, \\
0, & \alpha \pi \leq|\omega|<\pi,\end{cases} \\
& H_{1}(\omega)= \begin{cases}0, & |\omega| \leq(1-\beta), \\
\theta\left[\frac{\alpha \pi-\omega}{\alpha+\beta-1}\right], & (1-\beta) \pi \leq \omega<\alpha \pi, \\
1, & \alpha \pi \leq|\omega|<\pi .\end{cases}
\end{aligned}
$$

The maximum number of decomposition layers of TQWT can be obtained by

$$
J_{\max }=\left\lfloor\frac{\log (\beta N s / 8)}{\log (1 / \alpha)}\right\rfloor .
$$






(a)

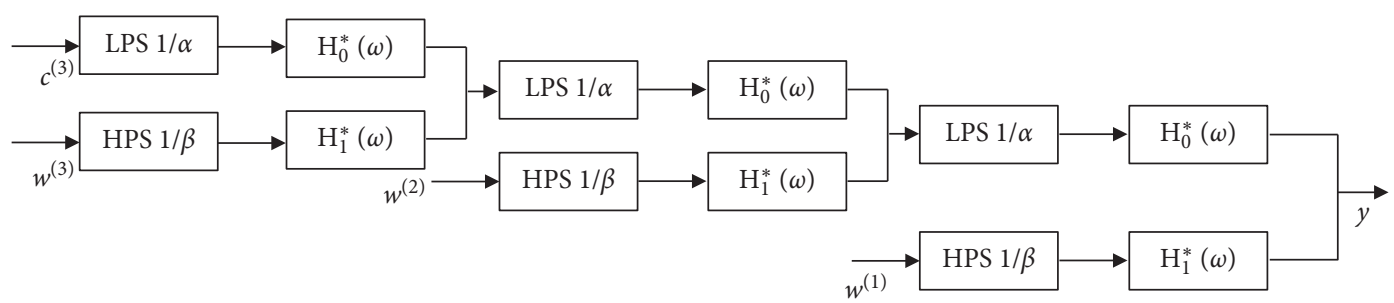

(b)

Figure 1: The corresponding dual channel filter banks of (a) decomposition process and (b) reconstruction process.

The scale factors $\alpha$ and $\beta$ can be substituted into equation (3) to obtain

$$
J_{\max }=\left\lfloor\frac{\log (N s /(4(Q+1)))}{\log [(Q+1) /(Q+1-(2 / r))]}\right\rfloor .
$$

In summary, the filtering characteristics of TQWT change with the Q-factor and the redundancy factor $r$. As the number of decomposition layers increases, the center frequency of the filter gradually decreases and the bandwidth also decreases. When the sampling frequency of the signal is $f_{s}$, the center frequency $f_{c}$ and bandwidth $B_{\omega}$ can be expressed as equations (5) and (6), respectively:

$$
\begin{aligned}
& f_{c}=\alpha^{j} \frac{2-\beta}{4 \alpha} f_{s}, \\
& B_{\omega}=\frac{1}{2} \beta \alpha^{(j-1)} \pi f_{s} .
\end{aligned}
$$

2.2. 1.5D Spectral Kurtosis. For a discrete sequence of signal $x(t)$, the system response is represented by $X(t)$, which can be expressed as

$$
X(t)=\int_{-\infty}^{+\infty} e^{2 \pi f j t} H(t, f) \mathrm{d} x(f),
$$

where $H(t, f)$ is the transfer function and $\mathrm{d} x(f)$ is the spectral increment.

The second-order spectral distance of $X(t)$ can be expressed as

$$
S_{2 n y}(t, f)=\frac{E\left\{\left|H(t, f) \mathrm{d} X(f)^{2 n}\right|\right\}}{\mathrm{d} f}=|H(t, f)|^{2 n} \cdot S_{2 n x} .
$$

When $2 n=2$, equation (8) can be abbreviated as

$$
S_{2 y}(t, f)=E\left\{\left|H(t, f)^{2}\right|\right\} \cdot \sigma_{x}^{2} .
$$

Furthermore, the fourth-order spectral cumulant can be expressed as

$$
C_{4 y}(f)=S_{4 y}(f)-2 S_{2 y}^{2}(f) .
$$

The spectral kurtosis can be defined by fourth-order normalized cumulants, as shown in

$$
\begin{aligned}
K_{x} & =\frac{C_{4 y}(\mathrm{f})}{S_{2 \mathrm{y}}^{2}(f)}=\frac{S_{4 y}(f)-2 S_{2 y}^{2}(f)}{S_{2 \mathrm{y}}^{2}(f)}=\frac{S_{4 y}(f)}{S_{2 \mathrm{y}}^{2}(f)}-2 \\
& =\frac{\left\langle|H(\mathrm{t}, f)|^{4}\right\rangle}{\left\langle|H(t, f)|^{2}\right\rangle^{2}}-2 .
\end{aligned}
$$

For a signal $x(t)$, the diagonal slice of the third-order cumulant $R_{3 x}\left(\tau_{1}, \tau_{2}\right)\left(\tau_{1}=\tau_{2}=\tau\right)$ can be expressed by

$$
R_{3 x}(\tau, \tau)=E\{x(t) x(t+\tau) x(t-\tau)\},
$$

where $E\{\cdot\}$ represents mathematical expectation.

The $1.5 \mathrm{D}$ spectrum of the original signal $x(t)$ can be obtained by performing Fourier transform on the three diagonal slices as

$$
B(\omega)=\int_{-\infty}^{\infty} R_{3 x}(\tau, \tau) e^{-j \omega \tau} \mathrm{d} \tau .
$$

The spectral distance of the spectrum discrete sequence is calculated and brought into equation (11), and the 1.5D spectral kurtosis value ${ }_{K}^{1.5 D}$ is obtained as

$$
K_{1.5 D}=\frac{\left\langle\left|B^{3}(t, f)\right|^{4}\right\rangle}{\left\langle\left|B^{3}(t, f)\right|^{2}\right\rangle^{2}}-2 .
$$

In order to compare the robustness of the kurtosis index defined in the time domain with the 1.5D spectral kurtosis index defined in the frequency domain against noise, different degrees of noise are added to the periodic impulse simulated signal. The mathematical model of the simulated signal can be expressed by 


$$
S(t)=\sum_{i=1}^{n} A e^{-\xi\left[t-q_{i}(t) / f_{\text {outer }}\right]^{2}} \cdot \sin \left(2 \pi f_{o} t\right)+n(t),
$$

where $\mathrm{A}$ is the amplitude, $\xi$ is the damping ratio, $f_{o}$ is the natural frequency of the system, $f_{\text {outer }}$ is the characteristic frequency, and $n(t)$ is the noise. The parameters of the simulated signal are listed in Table 1.

Figures 2(a)-2(p) are the time-domain waveforms and 1.5D spectrum of simulated signals with different SNR. Figure 2(q) shows the variation trend of the kurtosis index in the time domain and the $1.5 \mathrm{D}$ spectral kurtosis index in the frequency domain with the SNR. It can be seen from the figure that when the periodic impulse in the time-domain signal is gradually overwhelmed by noise, the spectral impact component composed of the fault characteristic frequency and its harmonics in the $1.5 \mathrm{D}$ spectrum is still obvious. This shows that the $1.5 \mathrm{D}$ spectrum has a relatively strong ability to suppress noise. By comparing the variation trends of the time-domain kurtosis index and the $1.5 \mathrm{D}$ spectral kurtosis index, it can also be found that when the SNR ratio is low, the 1.5D spectral kurtosis has a higher slope, indicating that it has a higher resolution.

Then, the following simulated signals (Figures 3(a) and 3(b)) are used to compare the robustness of the kurtosis index and the $1.5 \mathrm{D}$ spectral kurtosis index against nonperiodic transient impulse components. The time-domain kurtosis and 1.5D spectral kurtosis are calculated for Gaussian noise, and the results are 2.9587 and 12.1610, respectively. Then, a set of nonperiodic transient impulse are added at $0.3 \mathrm{~s}$ of the simulated signal, and the kurtosis and $1.5 \mathrm{D}$ spectral kurtosis values are 9.3163 and 11.9932 , respectively. The results show that the kurtosis index defined in the time domain is easily interfered by nonperiodic transient impulse, and the $1.5 \mathrm{D}$ spectral kurtosis value is more robust to nonperiodic transient impulse.

\subsection{Proposed Method}

TABle 1: Parameters of the simulated signal.

\begin{tabular}{lc}
\hline Parameter type & Parameter values \\
\hline Amplitude A & 2 \\
Natural frequency $f_{o}(\mathrm{~Hz})$ & 2100 \\
Sampling frequency $(\mathrm{Hz})$ & 8192 \\
Sampling points & 8192 \\
Fault frequency $(\mathrm{Hz})$ & 50 \\
\hline
\end{tabular}

(1) Firstly, the parameters of TQWT are selected. According to the characteristics of the frequency band where the bearing fault information is located, the number of signal decomposition layers $J$ is selected as 6 , which is beneficial to abandon the lowfrequency modulation harmonic characteristics and the high-frequency interference influence. The Q-factor is obtained through traversal optimization within a certain range, and this paper uses 0.5 as the step size to obtain the optimization between 2.5 and 5.

(2) The subbands under different Q-factors and decomposition scales are demodulated by Hilbert envelope, and the $1.5 \mathrm{D}$ spectrum of the demodulated signal is calculated, and then the $1.5 \mathrm{D}$ spectral kurtosis value is calculated for each discrete spectrum sequence.

(3) The optimal subband is selected, and its time-domain signal and the corresponding $1.5 \mathrm{D}$ spectrum are analyzed, which can extract fault feature information.

\section{Simulated Analysis}

In order to demonstrate the process of the proposed method and verify the effectiveness of the proposed method, a group of simulated signals are used for analysis. The mathematical model of simulated signal can be expressed by

$$
\left\{\begin{array}{l}
y_{1}=\sum_{i=1}^{n} A e^{-\xi\left[t-q_{i}(t) / f_{\text {outer }}\right]^{2}} \cdot \sin \left(2 \pi f_{o} t\right), \\
y_{2}=A_{1} e^{\left[-b_{1} \times\left(t-t_{1}\right)\right]} \cdot \sin \left[2 \pi f_{1} \cdot\left(t-t_{1}\right)\right]+A_{2} e^{\left[-b_{2} \times\left(t-t_{2}\right)\right]} \cdot \sin \left[2 \pi f_{2} \cdot\left(t-t_{2}\right)\right] \\
y_{3}=A_{3} \cdot \sin \left(2 \pi f_{\text {mesh }} t+\beta_{1}\right)\left(1+A_{4} \sin \left(2 \pi f_{h} t+\beta_{2}\right)\right), \\
Y=y_{1}+y_{2}+y_{3}+n(t) .
\end{array}\right.
$$

The simulated signal consists of four parts: $y_{1}$ represents periodic impulse components, $y_{2}$ represents nonperiodic transient impulse components, $y_{3}$ represents modulation harmonic components, $n(t)$ represents a certain degree of noise, and $Y$ is the composite signal of four components. Where $A=2$ is the amplitude of the periodic impulse components, $A_{1}=3$ and $A_{2}=6$ are the amplitudes of the nonperiodic transient impulse components, $A_{3}=A_{4}=0.6$ are the amplitudes of the modulation harmonic components. $\xi$ is the damping ratio, $\mathrm{f}_{\mathrm{o}}$ is the natural frequency of the system, and $\mathrm{f}_{\text {outer }}$ is the characteristic frequency of the periodic impulse components. $b_{1}=260, b_{2}=360, t_{1}=0.2, t_{2}=0.3$, and $f_{1}=f_{2}=$ $3600 \mathrm{~Hz}$ are damping parameters, time parameters, and natural frequencies of nonperiodic transient impulse components, respectively. $n(t)$ is the noise $(\mathrm{SNR}=-12 \mathrm{~dB})$. The parameters of the simulated signal are listed in Table 2. 




(a)

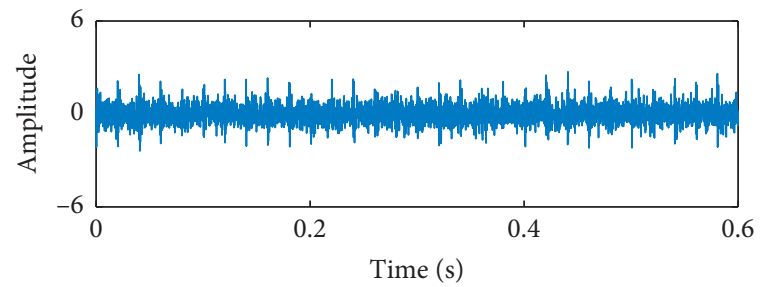

(c)

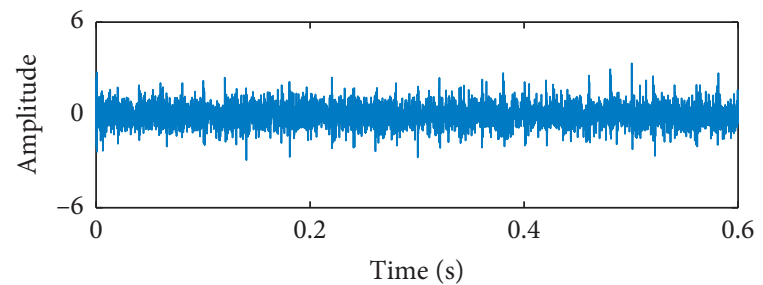

(e)

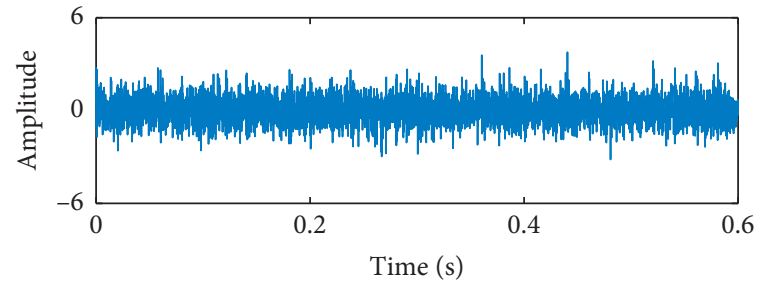

(g)

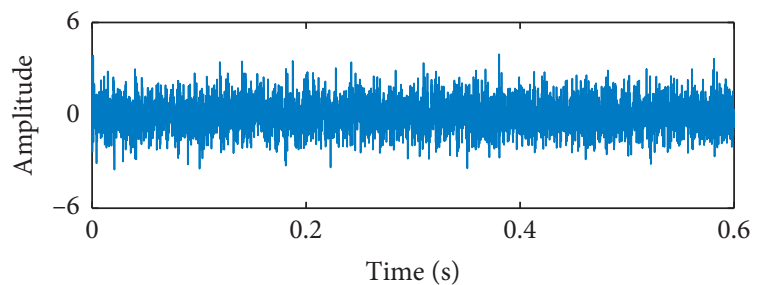

(i)

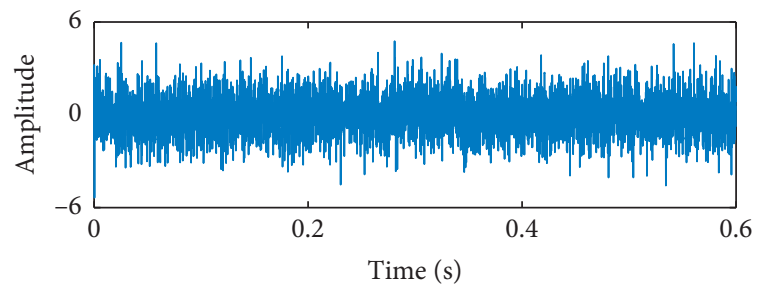

(k)

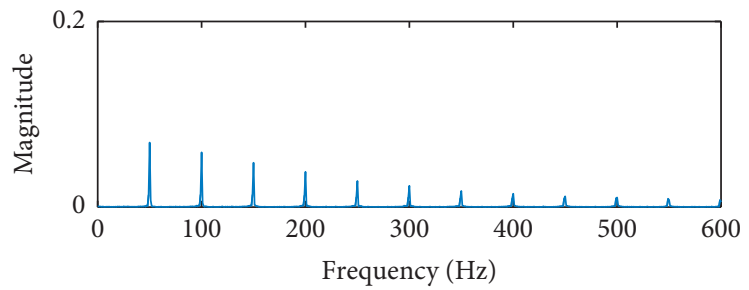

(b)



(d)



(f)

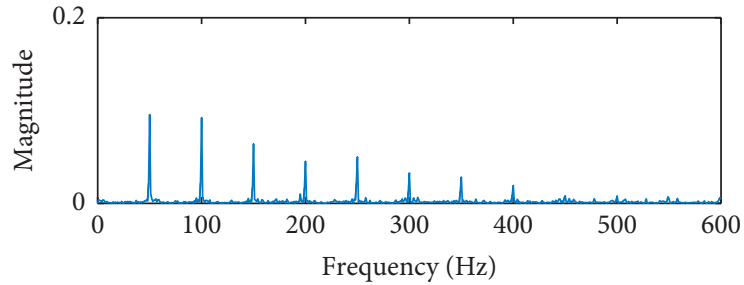

(h)



(j)

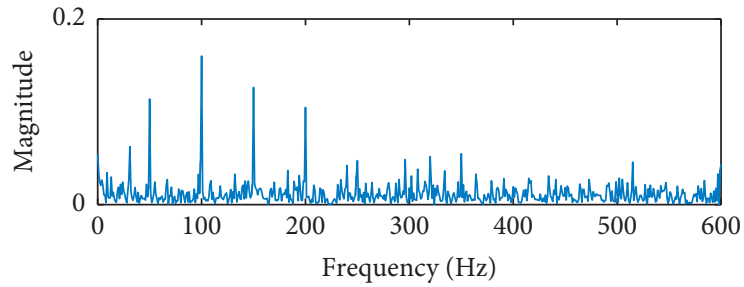

(1)

Figure 2: Continued. 


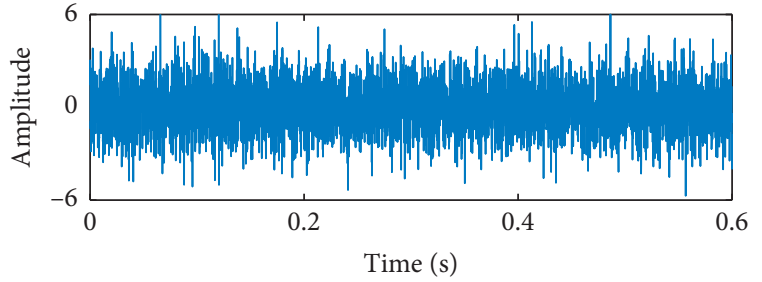

$(\mathrm{m})$

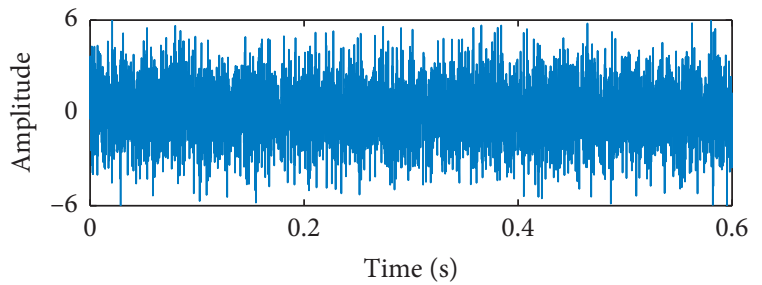

(o)

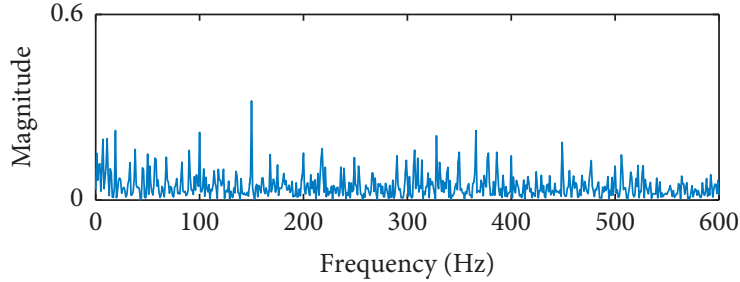

(n)

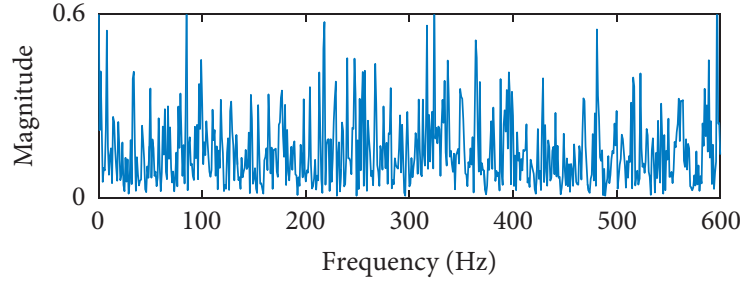

(p)

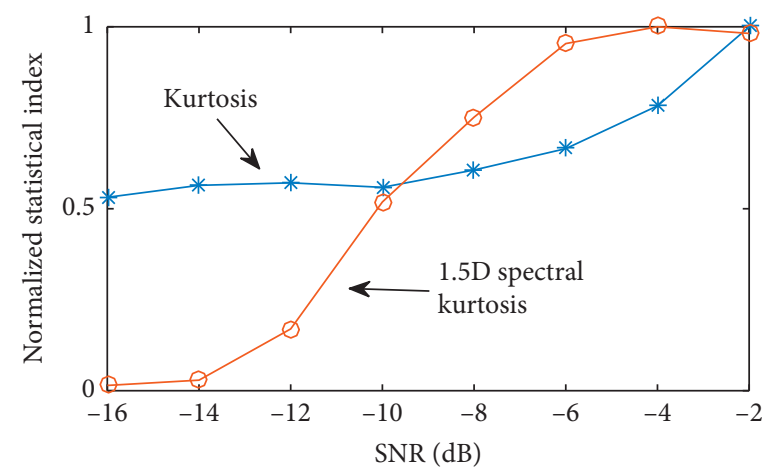

(q)

FIgure 2: Curve of the kurtosis and 1.5D spectral kurtosis with different SNR. (a) SNR $=-2 d B$. (b) SNR $=-2 d B$. (c) $\mathrm{SNR}=-4 d B$. (d) $\mathrm{SNR}=-4 d B$. (e) $\mathrm{SNR}=-6 d B$. (f) $\mathrm{SNR}=-6 d B$. (g) $\mathrm{SNR}=-8 d B$. (h) $\mathrm{SNR}=-8 d B$. (i) $\mathrm{SNR}=-10 d B$. (g) $\mathrm{SNR}=-10 d B$. (k) $\mathrm{SNR}=-12 d B$. (l) $\mathrm{SNR}=-12 d B$. (m) SNR $=-14 d B$. (n) SNR $=-14 d B$. (o) $\mathrm{SNR}=-16 d B .(\mathrm{p}) \mathrm{SNR}=-16 d B .(\mathrm{q}) \mathrm{SNR}(\mathrm{dB})$.

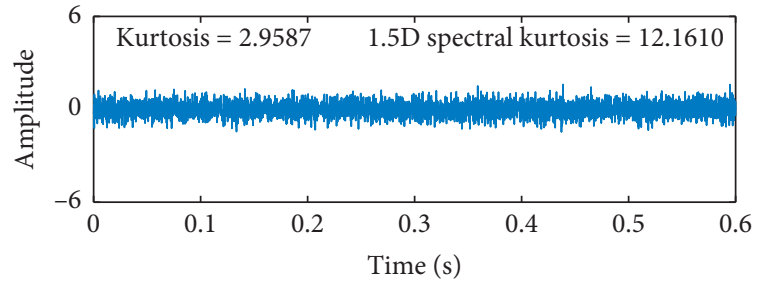

(a)

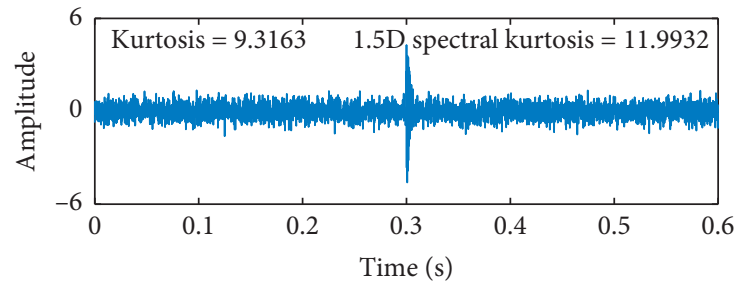

(b)

Figure 3: Analysis of simulated signals. (a) Gaussian noise; (b) noise accompanied by nonperiodic transient impulse.

TABle 2: Parameters of the simulated signal.

\begin{tabular}{lc}
\hline Parameter type & Parameter values $(\mathrm{Hz})$ \\
\hline Sampling frequency & 8192 \\
Natural frequency $f_{o}$ & 2100 \\
Natural frequency $f_{1}=f_{2}$ & 3600 \\
Carrier frequency $f_{\text {mesh }}$ & 360 \\
Modulation frequency $f_{h}$ & 15 \\
Fault frequency $f_{\text {outer }}$ & 50 \\
\hline
\end{tabular}

Figures 4(a)-4(e) show the time-domain waveforms of four components in the simulated signal, and they are periodic impulse components, nonperiodic transient impulse components, modulation harmonic components, and noise components, and Figure 4(e) shows the composite signal of four components.

The simulated signal is analyzed using the method proposed in this paper. By adjusting the adjustable factor $Q$, 


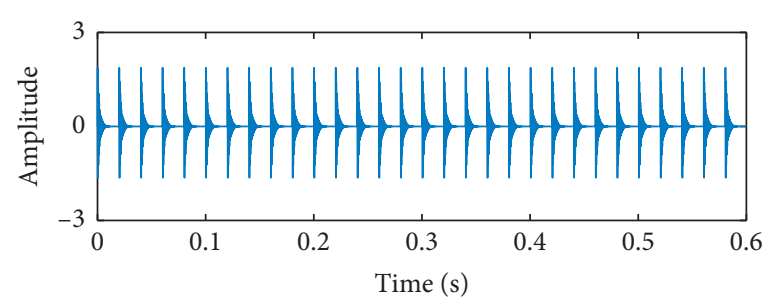

(a)

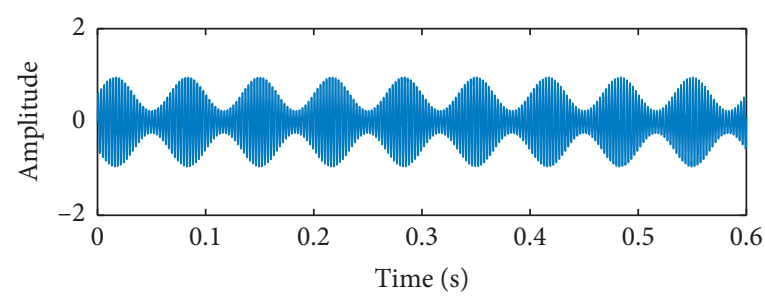

(c)

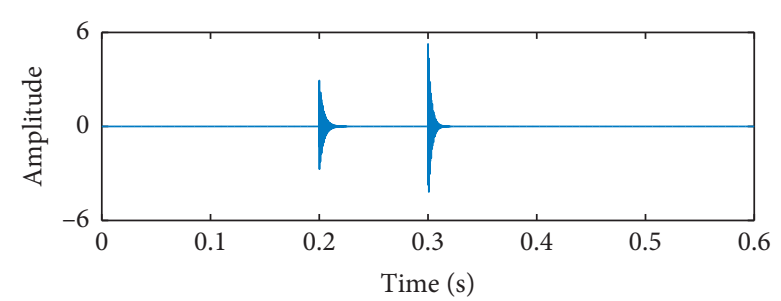

(b)

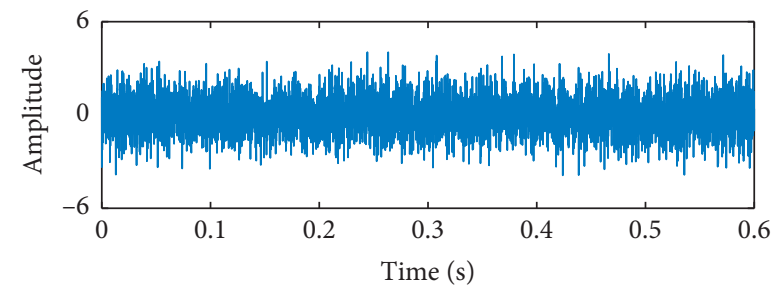

(d)

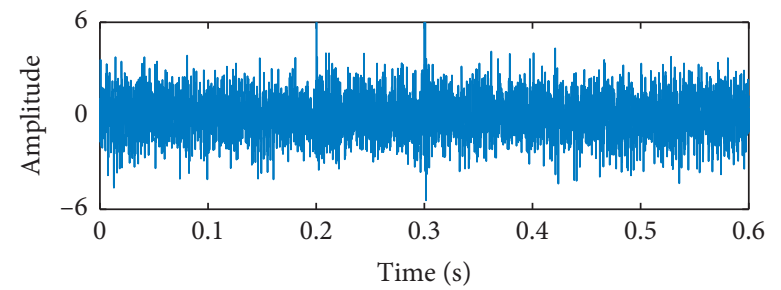

(e)

FIgURE 4: Time-domain waveform of simulated signals. (a) Periodic impulse components $\left(y_{1}\right)$, (b) nonperiodic transient impulse components $\left(y_{2}\right),(\mathrm{c})$ modulation harmonic components $\left(y_{3}\right),(\mathrm{d})$ noise components $(n(t))$, and (e) composite signal of four components $(Y)$.

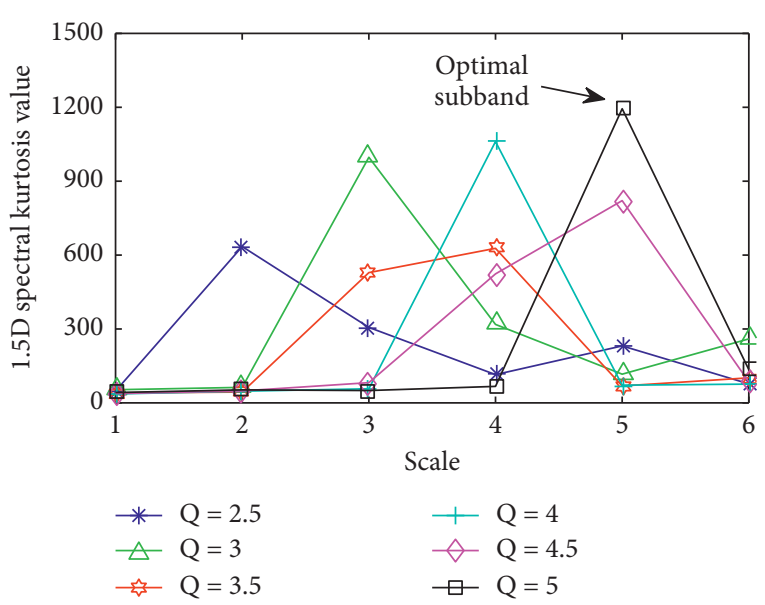

FIGURE 5: Optimal subband selection process of the 1.5D spectral kurtosis-guided TQWT method.

the TQWT with different sparsity are constructed, and then the $1.5 \mathrm{D}$ spectral kurtosis values corresponding to different wavelet subbands are calculated. The result is shown in Figure 5 . The horizontal axis represents different scales, the color of the line represents the Q-factor, and the vertical axis represents the $1.5 \mathrm{D}$ spectral kurtosis value. It can be seen that when the Q-factor is 5 and the scale is 5 , the corresponding subband index value reaches the maximum, indicating that the subband is the optimal subband.

The process of finding the optimal Q-factor and scale is equivalent to finding the optimal center frequency and bandwidth of the filter bank, which can be represented by the following equivalent filtering process (Figure 6).

The selected subband with Q-factor of 5 and scale of 5 is analyzed, and its time domain and $1.5 \mathrm{D}$ spectrum are shown in Figures 7(a) and 7(b). And the fault characteristic frequency and its multiplication component can be extracted.

For comparison, the kurtosis-guided TQWT method is used to select subbands. The optimal subband is corresponding to the Q-factor of 5 and scale of 1 (Figure 8(a)). The subband signal is analyzed (Figures $8(\mathrm{~b})$ and $8(\mathrm{c})$ )), and it can be found that the subband signal is the nonperiodic transient impulse components, and no fault characteristic information can be obtained.

\section{Experimental Analysis}

4.1. Experimental Data with Nonperiodic Transient Impulse Components. In order to further verify the effectiveness of the proposed method in processing bearing fault signals containing abnormal impulse components, the bearing vibration signal data of CWRU are analyzed. The experimental platform is shown in Figure 9. The bearing model used is JEMSKF6203-2RS. The selected experimental data are the rolling element fault data with relatively small fault size and 


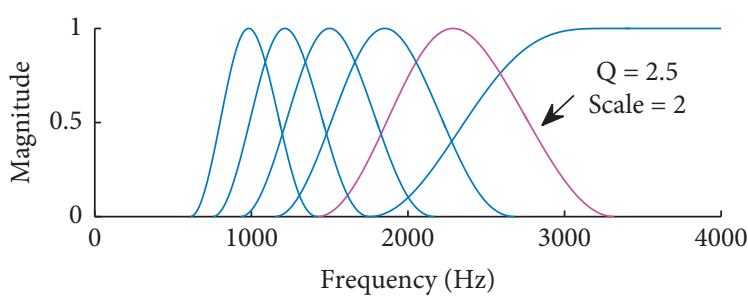

(a)

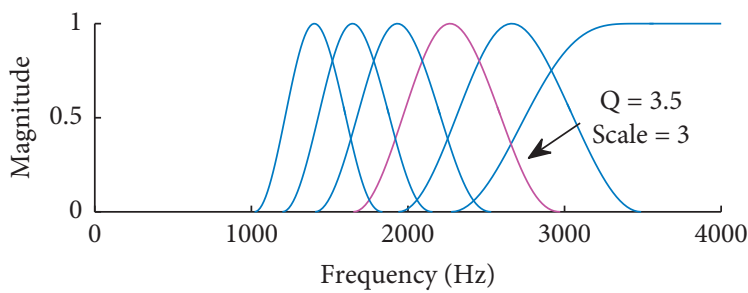

(c)

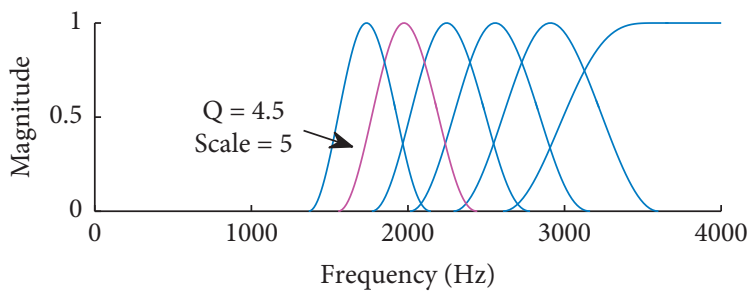

(e)

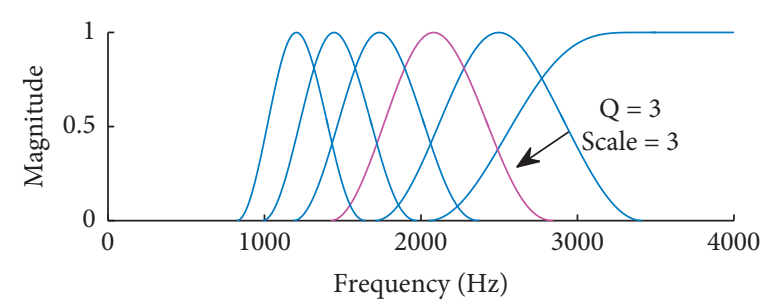

(b)

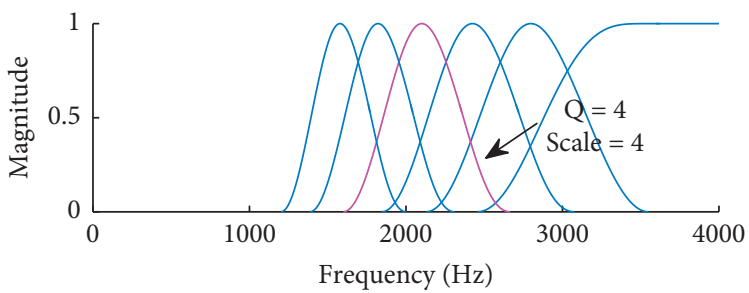

(d)

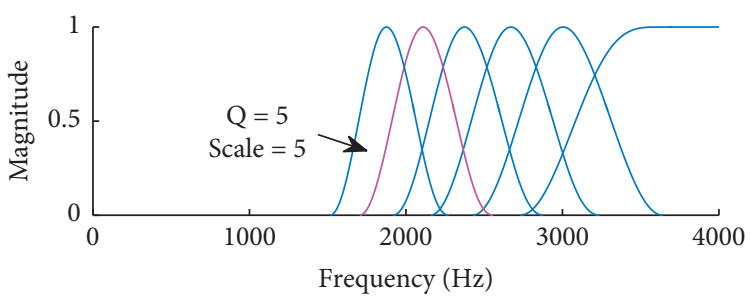

(f)

FIgURE 6: Equivalent filtering characteristics of subbands with different Q-values and scales.

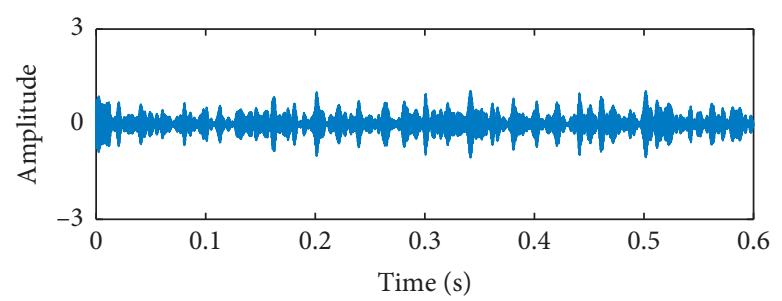

(a)

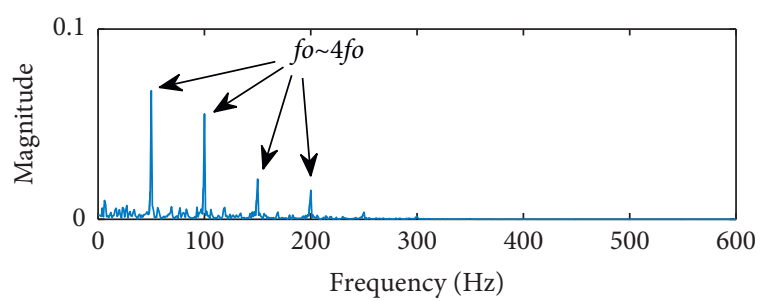

(b)

Figure 7: (a) Time-domain waveform and (b) 1.5D spectrum of the optimal subband.

weak fault degree. The driving motor speed is $1750 \mathrm{rpm}$, and the sampling frequency is $12000 \mathrm{~Hz}$. Through the above information, combined with the calculation equations of bearing fault characteristic frequency, the theoretical fault characteristic frequency under different types of bearing faults can be obtained, as shown in Table 3 .

The time-domain waveform of the intercepted experimental data is shown in Figure 10. It can be seen from the figure that this segment of data has obvious nonperiodic transient impulse components. The original signal is processed by the method proposed in this paper, and the results are shown in Figure 11. It can be seen from Figure 11(a) that the optimal subband is selected when the Q-factor is 5 and the scale is 2 . Then, the subband is analyzed. The time- domain waveform is shown in Figure 11(b), which contains obvious periodic impulse components. The $1.5 \mathrm{D}$ spectrum is shown in Figure 11(c). The characteristic frequency of rolling element fault is extracted. This shows that the proposed method is robust to the nonperiodic transient impulse components.

As a comparison, the kurtosis index is used to select the subband signals under different Q-factors and scales. The optimal subband is shown in Figure 12(a). The time-domain waveform of the subband is shown in Figure 12(b). It can be seen that its main component is nonperiodic transient impulse, and its $1.5 \mathrm{D}$ spectrum is shown in Figure 12(c), and no obvious fault feature information can be found. 


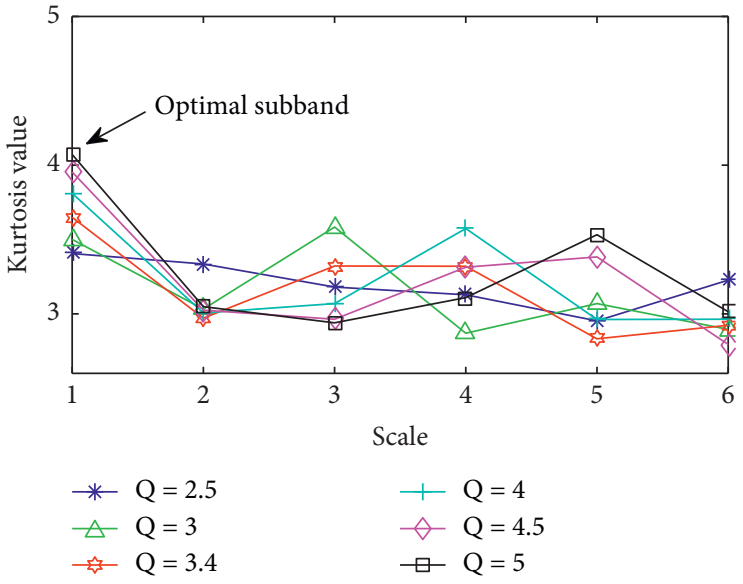

(a)
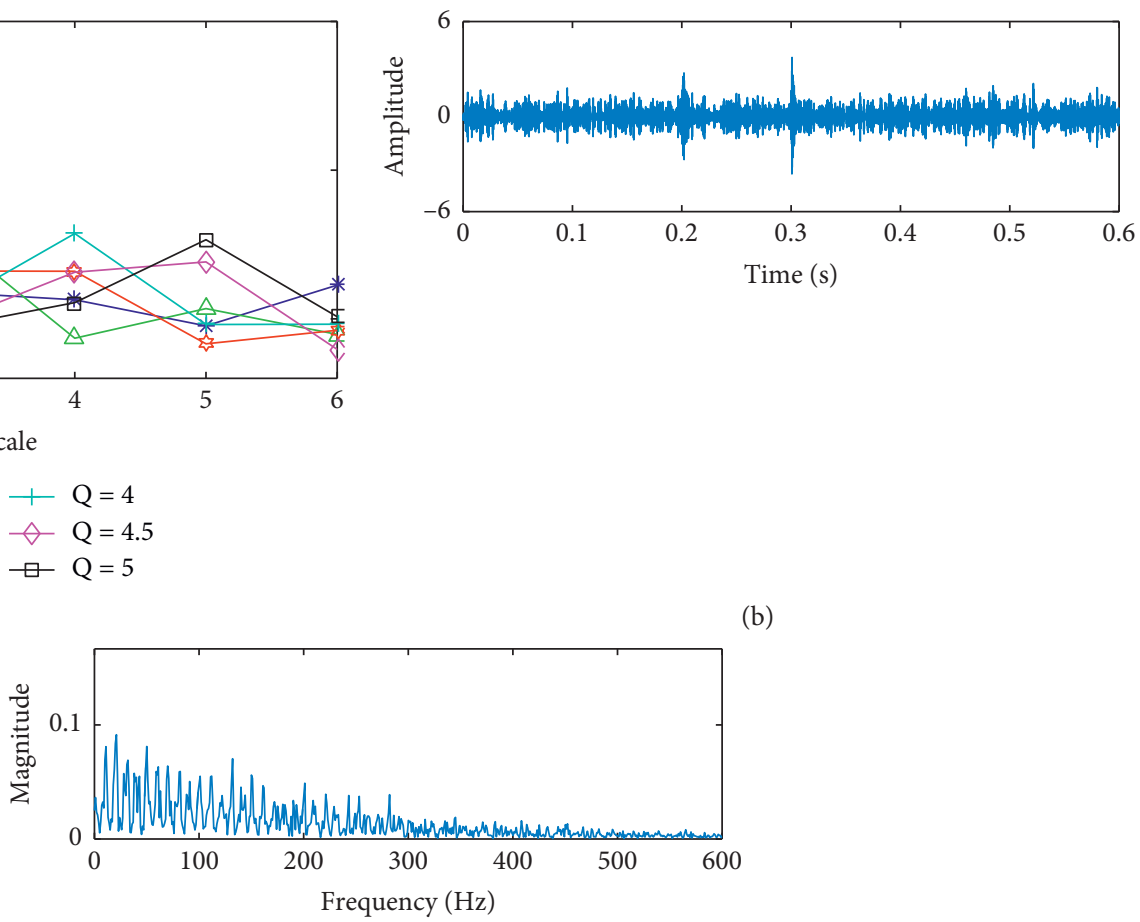

(b)

(c)

FIgURE 8: (a) Kurtosis-guided TQWT method, (b) time-domain waveform of the optimal subband, and (c) envelope spectrum of (b).

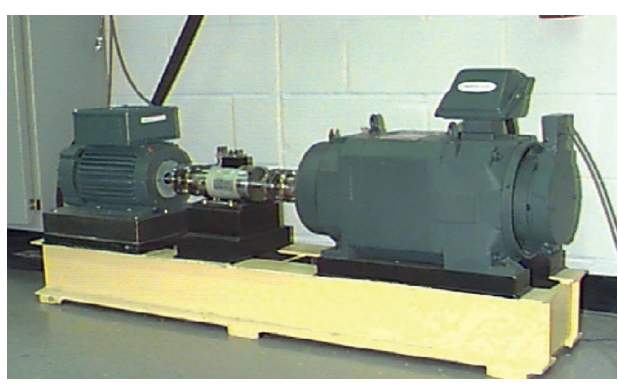

Figure 9: Bearing fault experimental equipment of CWRU.

TABLE 3: Theoretical fault characteristic frequency of bearings.

\begin{tabular}{lc}
\hline Type of fault & Parameter values $(\mathrm{Hz})$ \\
\hline Outer ring fault & 88 \\
Inner ring fault & 143 \\
Rolling element fault & 115 \\
\hline
\end{tabular}

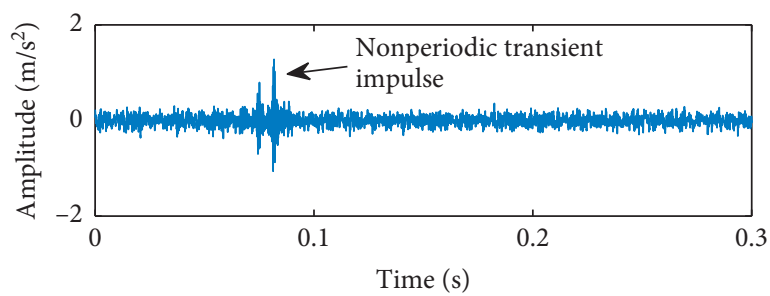

FIGURE 10: Time-domain waveform of the intercepted experimental data.
4.2. Experimental Data with Low Signal-to-Noise Ratio. In order to verify the processing ability of the proposed method for low signal-to-noise ratio signal, the data of QPZZ-II bearing test-bed are used for analysis and verification. The structure of the experimental platform is shown in Figure 13. The power source of the test-bed is the variable frequency drive motor. The motor is connected with the rotor through the coupling. The two ends of the rotor are bearing seats. The normal bearing is in the left bearing seat, and the fault bearing is in the right bearing seat. The structural parameters of the bearing are shown in Table 4 . The bearing model is 6205E. The speed of driving motor is $1470 \mathrm{rpm}$. The theoretical fault characteristic frequency under different types of bearing faults can be obtained, as shown in Table 5.

The time-domain waveform of the experimental signal is shown in Figure 14. It can be seen from the time-domain waveform that the signal-to-noise ratio of the vibration 


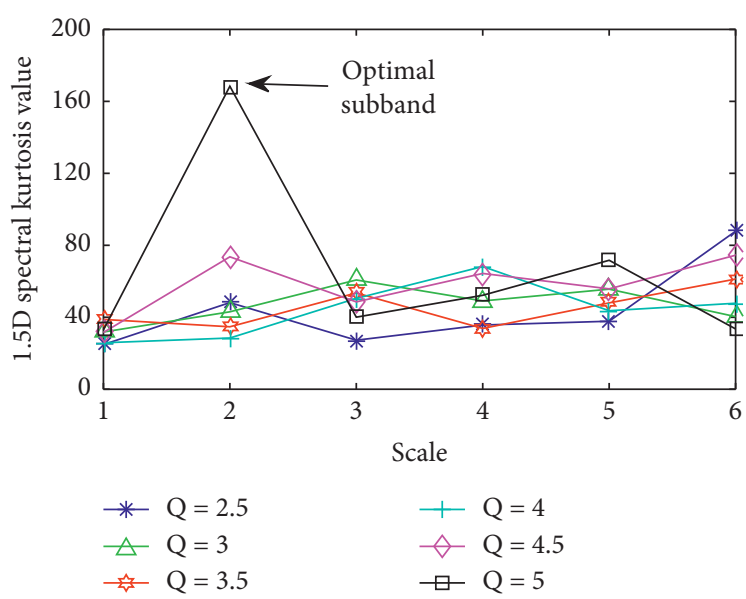

(a)
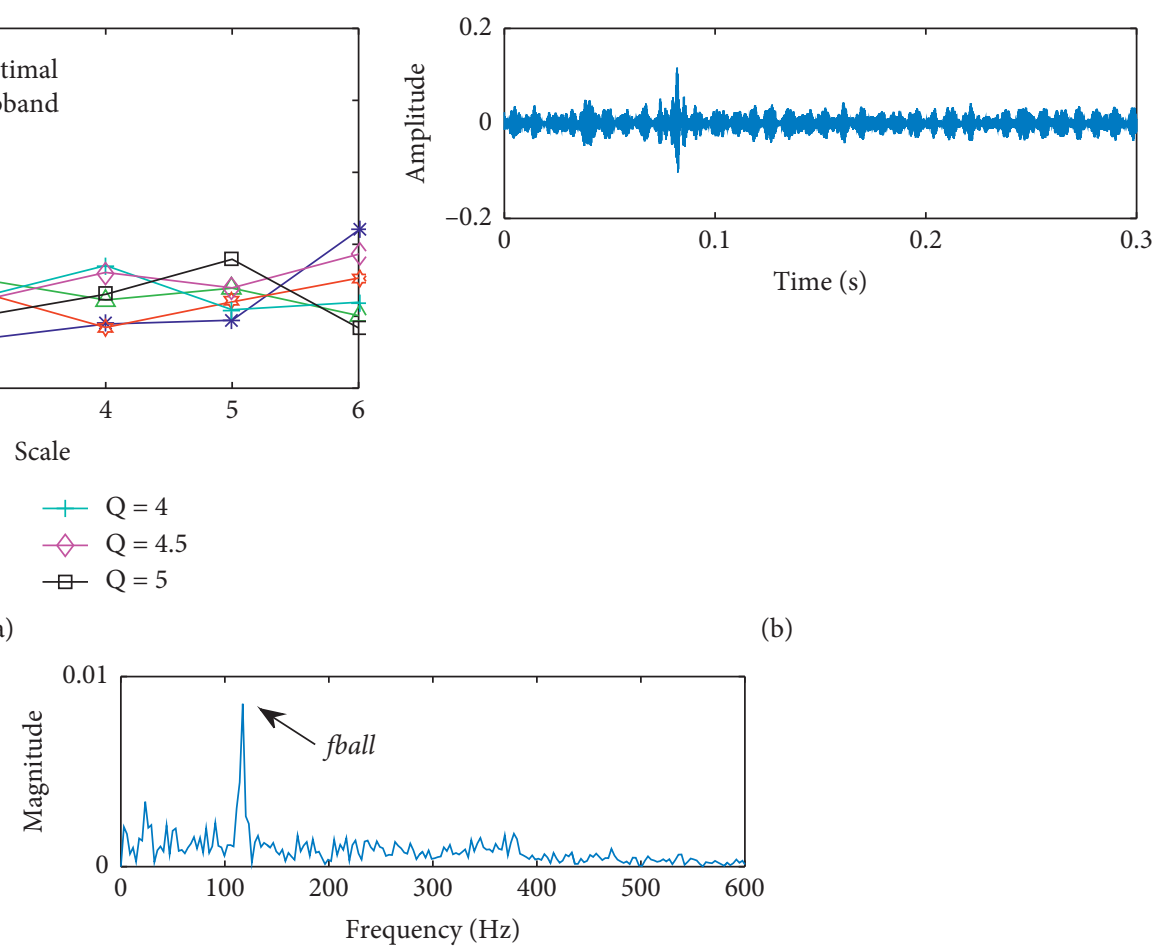

(c)

FIgURE 11: (a) Optimal subband selection process of the 1.5D spectral kurtosis-guided TQWT method, (b) time-domain waveform of the optimal subband, and (c) $1.5 \mathrm{D}$ spectrum of (b).

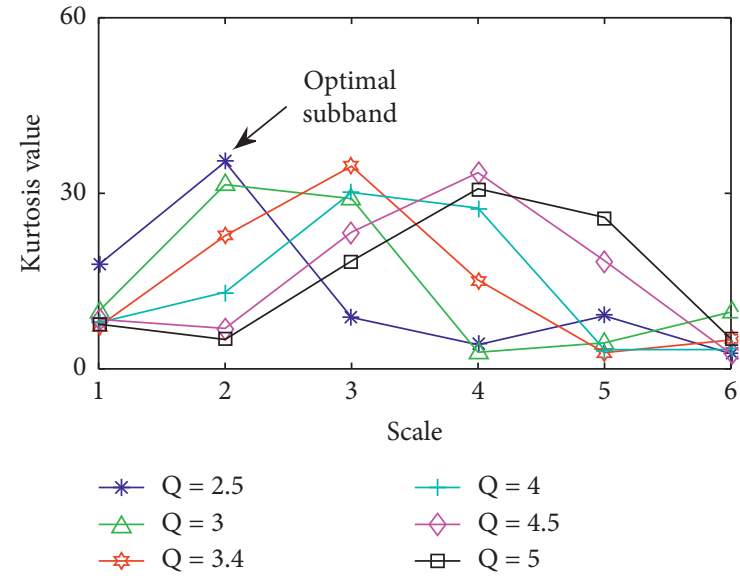

(a)

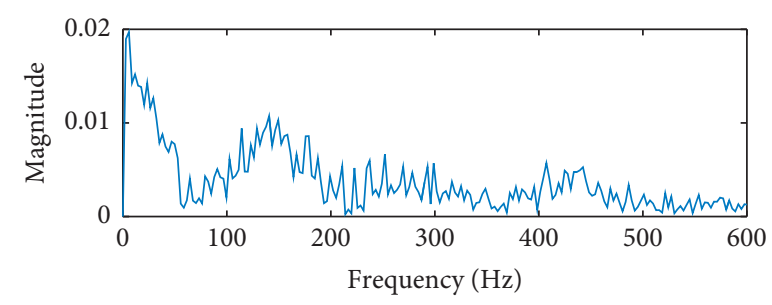

(c)

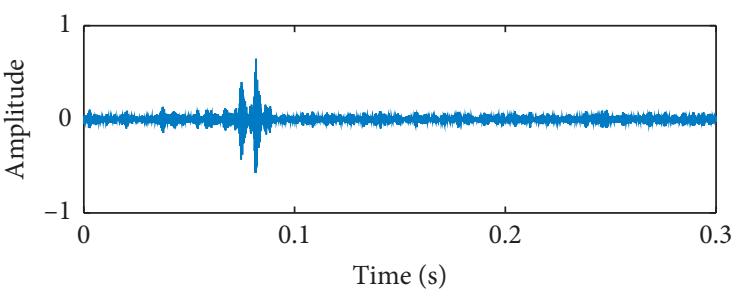

(b)

FIGURE 12: (a) Optimal subband selection process of the kurtosis-guided TQWT method, (b) time-domain waveform of the optimal subband, and (c) envelope spectrum of (b). 


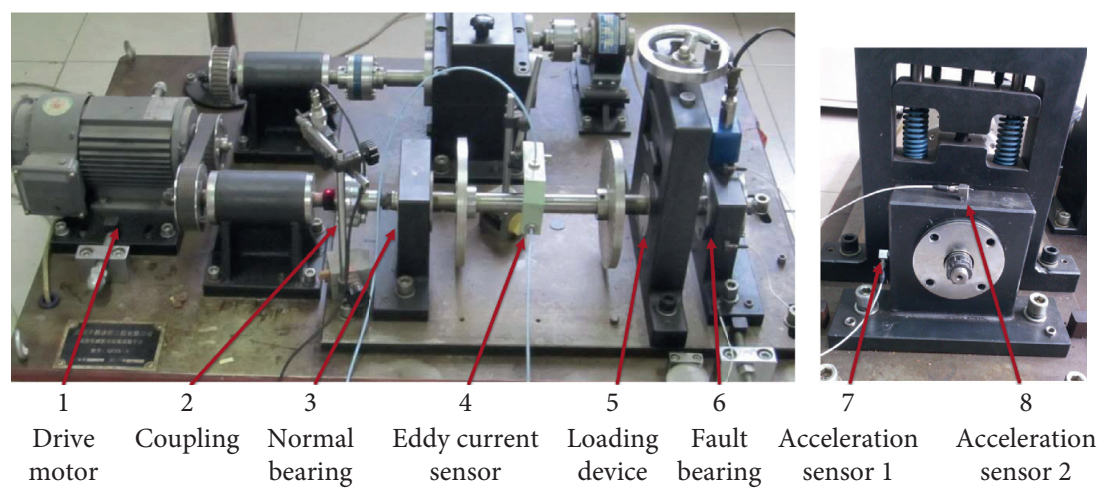

(a)

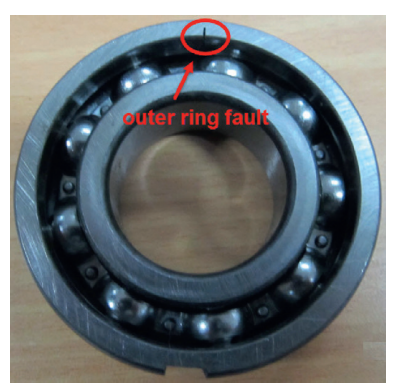

(b)

FIGURE 13: (a) Structure of the experimental platform; (b) bearing model of 6205E.

TABLE 4: Structural parameters of the bearing.

Parameter type

Parameter values

Inside diameter $(\mathrm{mm})$

25

Outside diameter $(\mathrm{mm})$

52

Ball diameter $(\mathrm{mm})$

Pitch diameter $(\mathrm{mm})$

39

Number of balls

Contact angle $\left(^{\circ}\right)$

TABLE 5: Theoretical fault characteristic frequency of bearings.

Type of fault

Parameter values $(\mathrm{Hz})$

Outer ring fault

Inner ring fault

132.6

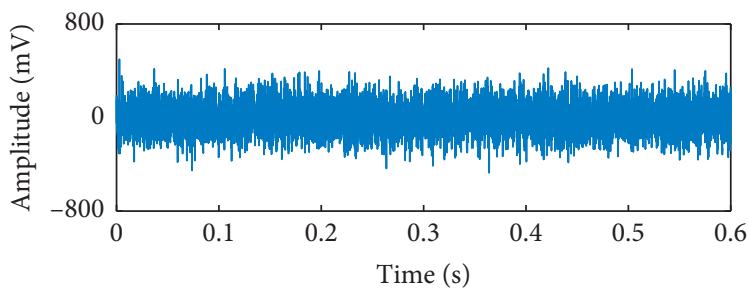

FIgURE 14: Time-domain waveform of the experimental data.

signal is low, and the periodic impulse characteristics are submerged. The original signal is analyzed by the kurtogram algorithm, and the analysis results are shown in Figure 15. It can be seen from Figure 15(a) that the optimal frequency band is in the low-frequency band (the frequency band is actually noise band, and the frequency band where the periodic impulse characteristics of bearing fault is located is about $2000 \mathrm{~Hz}$, which is submerged). The time-domain waveform and envelope spectrum of the optimal subband signal are shown in Figures 15(b) and 15(c), respectively. The method fails to extract the effective fault information.

The original signal is processed by the method proposed in this paper, and the results are shown in Figure 16. It can be seen from Figure 16(a) that the optimal subband is selected when the $\mathrm{Q}$-factor is 3 and the scale is 5 . Then, the subband is analyzed. The time-domain waveform is shown in Figure 16(b). The 1.5D 


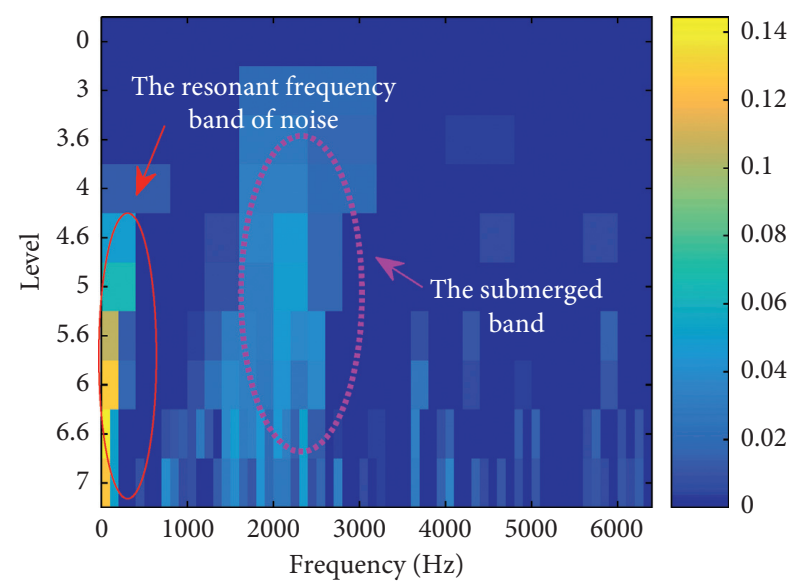

(a)

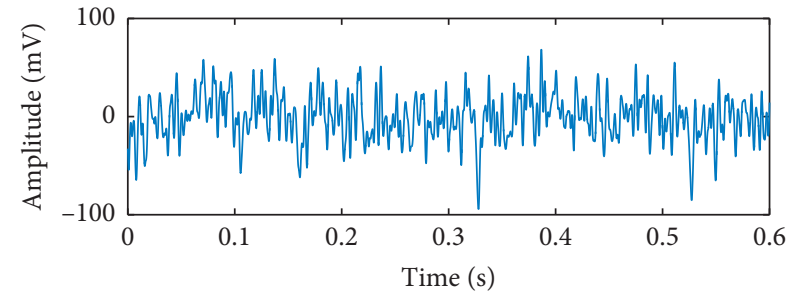

(b)

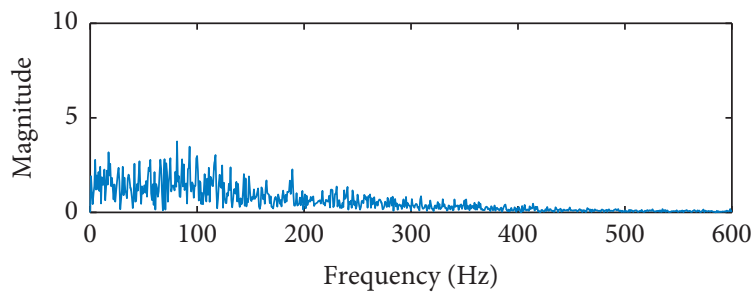

(c)

Figure 15: (a) Optimal subband selection process of the kurtogram method, (b) time-domain waveform of the optimal subband, and (c) envelope spectrum of (b).

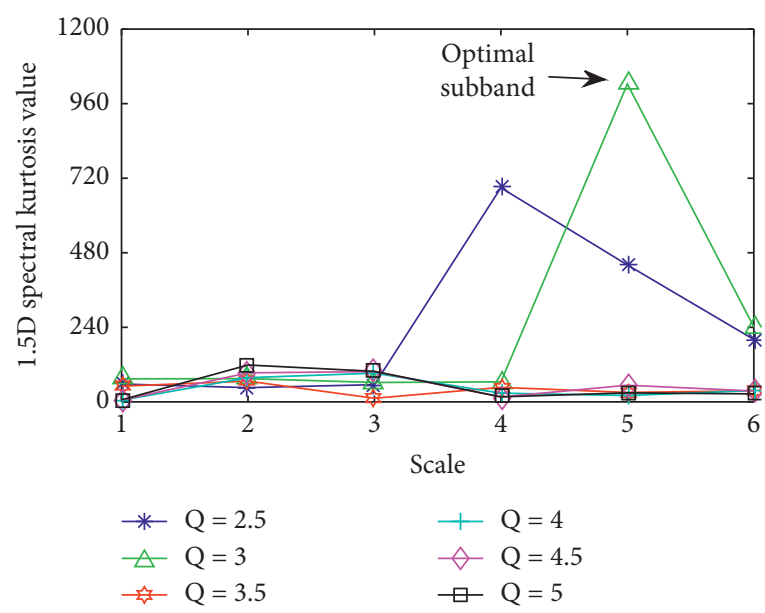

(a)

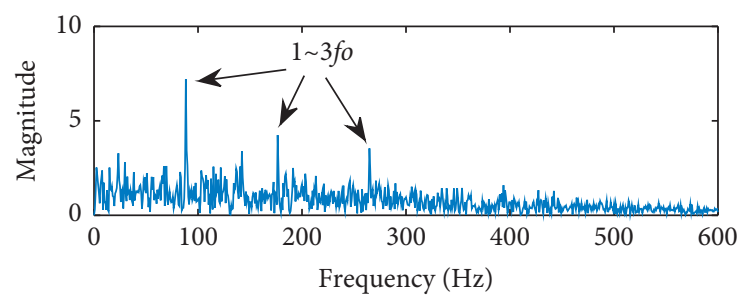

(c)

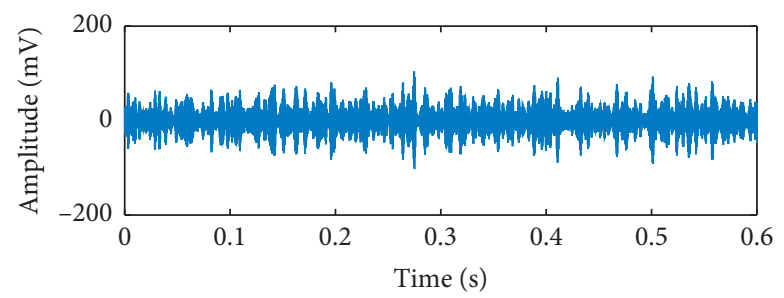

(b)

FIGURE 16: (a) Optimal subband selection process of the 1.5D spectral kurtosis-guided TQWT method, (b) time-domain waveform of the optimal subband, and (c) 1.5D spectrum of (b). 
spectrum is shown in Figure 16(c). The characteristic frequency of rolling element fault is extracted. This shows that the proposed method is robust to low signal-to-noise ratio signal.

\section{Conclusions}

The core problem in bearing signal processing is how to correctly select the frequency band of the fault information, so as to extract the information within the frequency band and filter out the interference information outside the frequency band. TQWT can provide a signal frequency band division method, and because its Q-factor is adjustable, it can adjust the wavelet oscillation characteristics to adapt to various signal characteristics. In order to improve the accuracy of subband selection and avoid the noise and nonperiodic transient impulse interference in the environment, this paper proposes a Q-factor and scale optimization method of TQWT with 1.5D spectral kurtosis as the evaluation index. Simulated and experimental results show that the proposed method has good analysis effect when SNR is low and when there is nonperiodic transient impulse interference.

\section{Data Availability}

The data used to support the findings of this study are available from the corresponding author upon request.

\section{Conflicts of Interest}

The authors declare no conflicts of interest.

\section{Authors' Contributions}

Xiong Zhang conceived and designed the experiments. Ming Zhang and Rujiang Hao performed the experiments. Xiong Zhang and Yuling He analyzed the data. Xiong Zhang and Shuting Wan contributed reagents/materials/analysis tools and wrote the paper.

\section{Acknowledgments}

This work was supported by the Fundamental Research Funds for the Central Universities (nos. 2020MS111 and 2018QN093) and the National Natural Science Foundation of China (no. 51777075).

\section{References}

[1] X. Liu, H. Huang, and J. Xiang, "A personalized diagnosis method to detect faults in a bearing based on acceleration sensors and an FEM simulation driving support vector machine," Sensors, vol. 20, no. 2, 2020.

[2] X. Wang and F. Liu, "Triplet loss guided adversarial domain adaptation for bearing fault diagnosis," Sensors, vol. 20, no. 1, 2020.

[3] H. Li, J. Huang, and S. Ji, "bearing fault diagnosis with a feature fusion method based on an ensemble convolutional neural network and deep neural network," Sensors, vol. 19, no. 9, 2019.
[4] Y. Peng, Z. Li, K. He, Y. Liu, Q. Li, and L. Liu, "Broadband mode decomposition and its application to the quality evaluation of welding inverter power source signals," IEEE Transactions on Industrial Electronics, vol. 67, no. 11, pp. 9734-9746, 2019.

[5] Y. Peng, Z. Wang, K. He et al., "Modulated broadband mode decomposition for the feature extraction of double pulse metal inert gas welding," IEEE Access, vol. 8, pp. 134031-134041, 2019.

[6] Y. Peng, Z. Wang, K. He et al., "Feature extraction of double pulse metal inert gas welding based on broadband mode decomposition and locality preserving projection," Mathematical Problems in Engineering, vol. 2020, Article ID 7576034, 16 pages, 2020.

[7] W. Deng, J. Xu, Y. Song et al., "Differential evolution algorithm with wavelet basis function and optimal mutation strategy for complex optimization problem," Applied Soft Computing, vol. 100, pp. 1-16, 2021.

[8] Y. Song, D. Wu, A. Wagdy Mohamed, X. Zhou, B. Zhang, and W. Deng, "Enhanced success history adaptive DE for parameter optimization of photovoltaic models," Complexity, vol. 2021, Article ID 6660115, 22 pages, 2021.

[9] W. Deng, J. Xu, H. Zhao, and Y. Song, "A novel gate resource allocation method using improved PSO-based QEA," IEEE Transactions on Intelligent Transportation Systems, pp. 1-9. In press, 2020.

[10] J. Antoni, "Fast computation of the kurtogram for the detection of transient faults," Mechanical Systems and Signal Processing, vol. 21, no. 1, pp. 108-124, 2005.

[11] Y. Lei, J. Lin, Z. He, and Y. Zi, "Application of an improved kurtogram method for fault diagnosis of rolling element bearings," Mechanical Systems and Signal Processing, vol. 25, no. 5, pp. 1738-1749, 2011.

[12] D. Wang and K.-L. Tsui, "Dynamic Bayesian wavelet transform: new methodology for extraction of repetitive transients," Mechanical Systems and Signal Processing, vol. 88, no. 5, pp. 137-144, 2017.

[13] Y. Qin, Y. Mao, B. Tang et al., "M-band flexible wavelet transform and its application to the fault diagnosis of planetary gear transmission systems," Mechanical Systems and Signal Processing, vol. 134, pp. 1-26, 2019.

[14] Y. Qin, J. Zou, B. Tang, Y. Wang, and H. Chen, "Transient feature extraction by the improved orthogonal matching pursuit and K-svd algorithm with adaptive transient dictionary," IEEE Transactions on Industrial Informatics, vol. 16, no. 1, pp. 215-227, 2020.

[15] J. Antoni, "The infogram: entropic evidence of the signature of repetitive transients," Mechanical Systems and Signal Processing, vol. 74, no. 6, pp. 73-94, 2016.

[16] C. Li, D. Cabrera, J. V. de Oliveira, R.-V. Sanchez, M. Cerrada, and G. Zurita, "Extracting repetitive transients for rotating machinery diagnosis using multiscale clustered grey infogram," Mechanical Systems and Signal Processing, vol. 76-77, no. 8, pp. 157-173, 2016.

[17] A. Moshrefzadeh and A. Fasana, "The Autogram: an effective approach for selecting the optimal demodulation band in rolling element bearings diagnosis," Mechanical Systems and Signal Processing, vol. 105, no. 8, pp. 294-318, 2018.

[18] I. W. Selesnick, "Wavelet transform with tunable Q-factor," IEEE Transactions on Signal Processing, vol. 59, no. 8, pp. 3560-3575, 2011.

[19] J. Zhao, Y. Zhang, and Q. Chen, "Rolling bearing fault feature extraction based on adaptive tunable Q-factor wavelet 
transform and spectral kurtosis," Shock and Vibration, vol. 2020, pp. 3560-3575, Article ID 8875179, 2020.

[20] P. Ma, H. Zhang, W. Fan, and C. Wang, "Early fault diagnosis of bearing based on frequency band extraction and improved tunable Q-factor wavelet transform," Measurement, vol. 137, pp. 189-202, 2019.

[21] J. Ding, J. Zhou, and Y. Yin, "Fault detection and diagnosis of a wheelset-bearing system using a multi-Q-factor and multilevel tunable Q-factor wavelet transform," Measurement, vol. 143, pp. 112-124, 2019. 